\title{
ARTICLE OPEN Mucins trigger dispersal of Pseudomonas aeruginosa biofilms
}

\author{
Julia Y. Co ${ }^{1,2}$, Gerardo Cárcamo-Oyarce ${ }^{3}$, Nicole Billings ${ }^{1}$, Kelsey M. Wheeler ${ }^{1,2}$, Scott C. Grindy ${ }^{4}$, Niels Holten-Andersen ${ }^{4}$ and \\ Katharina Ribbeck ${ }^{1}$
}

Mucus is a biological gel that lines all wet epithelia in the body, including the mouth, lungs, and digestive tract, and has evolved to protect the body from pathogenic infection. However, microbial pathogenesis is often studied in mucus-free environments that lack the geometric constraints and microbial interactions in physiological three-dimensional mucus gels. We developed fluid-flow and static test systems based on purified mucin polymers, the major gel-forming constituents of the mucus barrier, to understand how the mucus barrier influences bacterial virulence, particularly the integrity of Pseudomonas aeruginosa biofilms, which can become resistant to immune clearance and antimicrobial agents. We found that mucins separate the cells in $P$. aeruginosa biofilms and disperse them into suspension. Other viscous polymer solutions did not match the biofilm disruption caused by mucins, suggesting that mucin-specific properties mediate the phenomenon. Cellular dispersion depended on functional flagella, indicating a role for swimming motility. Taken together, our observations support a model in which host mucins are key players in the regulation of microbial virulence. These mucins should be considered in studies of mucosal pathogenesis and during the development of novel strategies to treat biofilms.

npj Biofilms and Microbiomes (2018)4:23; doi:10.1038/s41522-018-0067-0

\section{INTRODUCTION}

Human-associated bacteria often exist as biofilms, structured communities that secrete and encase themselves within a protective matrix. ${ }^{1-3}$ The healthy body must maintain homeostasis with these microbial communities; if growth is unchecked, then biofilms can lead to morbidities such as chronic and nosocomial infections. ${ }^{4-7}$ Mucus is a biological hydrogel that coats all wet epithelia in the body and forms a major ecological niche for the human microbiota, likely playing an important role in regulating host-microbe interactions. ${ }^{8-11}$

The gel-forming biopolymers that comprise mucus, called mucins, form bottlebrush-like structures with dense O-linked glycosylation ${ }^{12,13}$ and are important in maintaining health. Mucin dysregulation is associated with diseases such as cystic fibro$\mathrm{sis}^{14,15}$ chronic obstructive pulmonary disorder, ${ }^{16}$ and ulcerative colitis, ${ }^{17,18}$ and can also lead to pathogen overgrowth. ${ }^{14-18}$ Furthermore, mucins can promote clearance of microbes. ${ }^{19,20}$ Mucins impair the surface attachment and formation of biofilms by Streptococcus mutans, Candida albicans, ${ }^{21}$ and Pseudomonas aeruginosa ${ }_{i}^{22}$ surface attachment by these microbes can inflict damage on the host. Mucins also regulate the motility of Helicobacter pylori ${ }^{23}$ and suppress hyphae formation in $C$. albicans. $^{21}$ Despite the importance of mucins in regulating pathogens, microbial physiology in these mucosal systems is often studied in mucus-free environments, which lack the biochemistry and geometric constraints found in physiological, three-dimensional mucus gels.

To close this gap, here we explored whether mucin polymers destabilize biofilms of the opportunistic pathogen $P$. aeruginosa. Biofilms of $P$. aeruginosa can become resistant to immune clearance and antimicrobial agents, threatening human health. ${ }^{24-26}$ We used natively purified mucins for this study because they form viscoelastic hydrogels, in contrast to the commercially available mucins that lose this ability during the purification process. ${ }^{27,28}$ We discovered that natively purified mucins induce the dispersal of $P$. aeruginosa biofilms, suggesting that mucins are important regulators of microbial virulence.

\section{RESULTS}

Mucins disassemble $P$. aeruginosa biofilms

To determine whether mucins affect the integrity of established biofilms, $P$. aeruginosa cells were grown in a previously characterized flow-cell system. ${ }^{29}$ This flow-cell system permits continuous replenishment of mucins, thereby preventing changes in mucin availability due to degradation or adsorption to biofilm or flow-cell surfaces. Once matured into biofilms, $P$. aeruginosa cells were exposed to mucins and the resulting effects on the biofilms were analyzed using live confocal microscopy. We used natively purified MUC5AC, the primary secreted mucin found in the lungs and stomach. ${ }^{14}$ Biofilms of $P$. aeruginosa PAO1 tagged with GFP (PAO1-GFP) were grown under continuous flow at $0.5 \mu \mathrm{L} / \mathrm{min}$ for $48 \mathrm{~h}$, at which point they exhibited a relatively smooth, flat architecture (Fig. 1a). These biofilms were exposed to Luria Broth (LB) medium alone or to medium supplemented with $0.05,0.1,0.5$, or $1.0 \%(\mathrm{w} / \mathrm{v})$ mucins under continuous flow at $0.5 \mu \mathrm{L} / \mathrm{min}$ for $1 \mathrm{~h}$, and the extent of biofilm disruption was assessed. Treatment with 0.05 and $0.1 \%$ mucins did not disassemble the biofilms, while exposure to 0.5 or $1 \%$ mucins resulted in biofilm fragmentation and disassembly (Fig. 1a, b).

\footnotetext{
'Department of Biological Engineering, Massachusetts Institute of Technology, Cambridge, MA, USA; ${ }^{2}$ Microbiology Graduate Program, Massachusetts Institute of Technology, Cambridge, MA, USA; ${ }^{3}$ Research Laboratory of Electronics, Massachusetts Institute of Technology, Cambridge, MA, USA and ${ }^{4}$ Department of Materials Science and Engineering, Massachusetts Institute of Technology, Cambridge, MA, USA

Correspondence: Katharina Ribbeck (ribbeck@mit.edu)

These authors contributed equally: Julia Y. Co, Gerardo Cárcamo-Oyarce.
}

Received: 9 March 2018 Revised: 20 July 2018 Accepted: 4 September 2018

Published online: 10 October 2018 
a

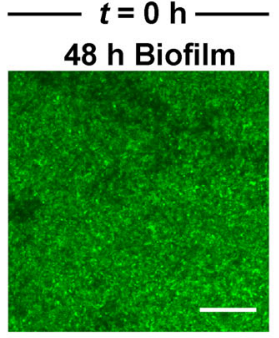

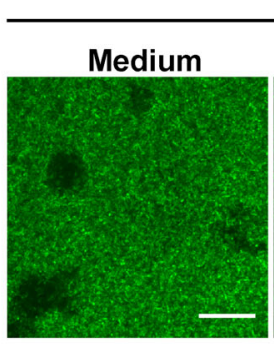

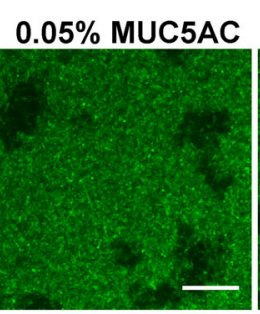
$\boldsymbol{t}=1 \mathrm{~h}$ Exposure

b

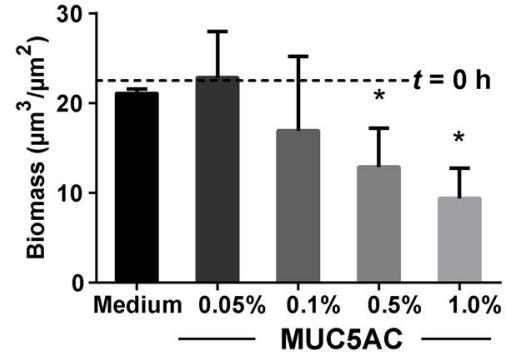

C

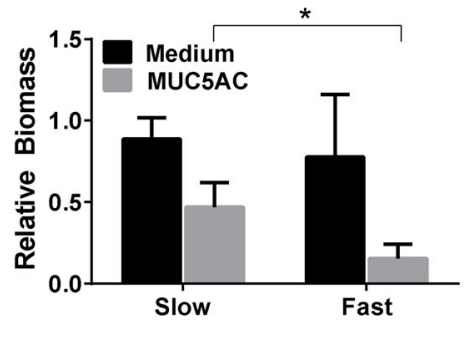

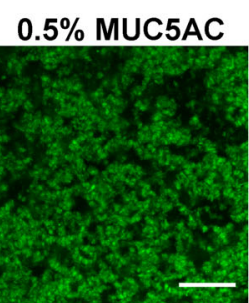

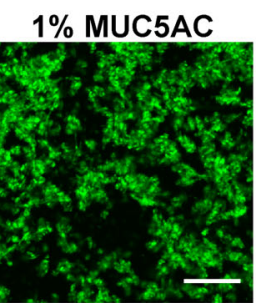

e

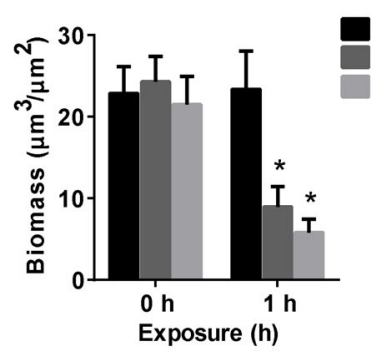

Medium MUC5AC MUC2 d

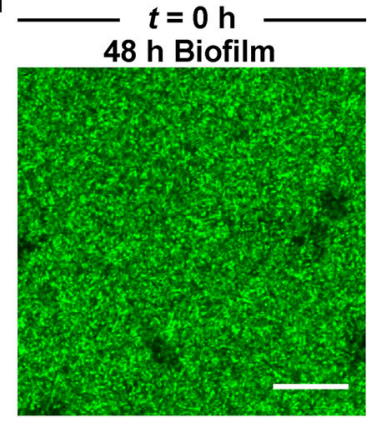

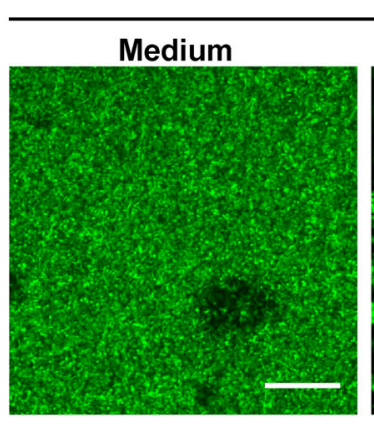

$t=1 \mathrm{~h}$ Exposure MUC5AC

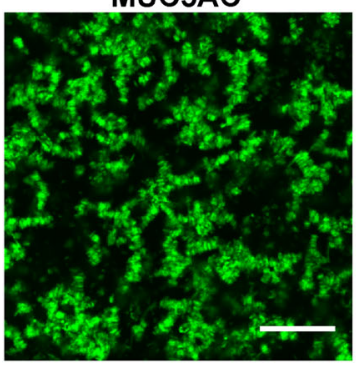

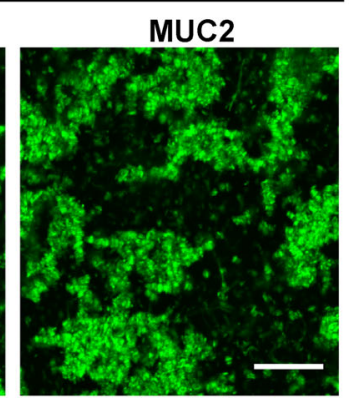

Fig. 1 Natively purified mucins MUC5AC and MUC2 trigger the disruption of $P$. aeruginosa biofilms. a PAO1-GFP biofilms (48 h) were exposed to medium with increasing concentrations of mucins. The flow rate of the medium was $0.5 \mu \mathrm{L} / \mathrm{min}$. At $0.5 \%$ ( $\mathrm{w} / \mathrm{v}$ ) and above, MUC5AC reduced biofilm mass. Scale bars $=20 \mu \mathrm{m}$. b Confocal images of biofilms were analyzed using COMSTAT to quantify biomass after $1 \mathrm{~h}$ of exposure to mucins. Dotted lines indicate average values for $48 \mathrm{~h}$ biofilms before exposure $(t=0 \mathrm{~h})$. Error bars represent standard error $(n \geq 3)$. * $P \leq 0.05$, unpaired Student's $t$ test. c Mucin-mediated biofilm erosion is affected by the flow rate. Quantification of $48 \mathrm{~h}$ PAO1-GFP biofilm biomass after $1 \mathrm{~h}$ of exposure to LB with or without $0.5 \%$ mucins at slow flow $(0.5 \mu \mathrm{L} / \mathrm{min})$ or fast flow $(10 \mu \mathrm{L} / \mathrm{min})$. Values are normalized to biofilm biomass before exposure. Error bars represent standard error $(n \geq 3)$. ${ }^{*} P \leq 0.05$, unpaired Student's $t$ test. A comparison between mucin MUC5AC and MUC2 shows that both mucins disassemble $P$. aeruginosa biofilms. $\mathbf{d}$ Live confocal imaging and e biofilm biomass quantification of $48 \mathrm{~h}$ PAO1GFP biofilms before $(t=0 \mathrm{~h})$ and after $(t=1 \mathrm{~h})$ exposure to medium with or without $0.5 \%$ mucins at $0.5 \mu \mathrm{L} / \mathrm{min}$ flow. Scale bars $=20 \mu \mathrm{m}$. Error bars represent standard error $(n=3) .{ }^{*} P \leq 0.05$, unpaired Student's $t$ test

These data indicate that mucins effectively disrupt biofilms above a threshold mucin concentration. Based on these results, subsequent analyses were performed using mucins at a concentration of $0.5 \%$.

Since these experiments were performed under fluid flow, the fluid mechanics (such as shear stress, which is dictated by solution viscosity and flow rate) of the system may influence biofilm growth and disassembly. ${ }^{30-32}$ To explore whether mucinmediated biofilm disruption is affected by flow rate, $48 \mathrm{~h}$ PAO1GFP biofilms were treated with or without $0.5 \%$ mucins for $1 \mathrm{~h}$ at slow flow $(0.5 \mu \mathrm{L} / \mathrm{min})$ or fast flow $(10 \mu \mathrm{L} / \mathrm{min})$. While increasing the flow of mucin-free LB did not result in substantial biofilm disassembly, fast-flow mucin treatment enhanced biofilm disassembly (Fig. 1c), resulting in biofilms with $31.6 \%$ less biomass than those treated via slow flow (Fig. 1c). Thus, a faster flow rate enhances mucin-mediated disassembly of $P$. aeruginosa biofilms.

To address whether the ability to disrupt biofilms was specific to MUC5AC, we repeated the experiment with native purified MUC2 from porcine intestinal mucus. As with exposure to MUC5AC, exposure to MUC2 for $1 \mathrm{~h}$ notably fragmented the biofilms (Fig.
$1 d$, e). Confocal images of the biofilms revealed that exposure to MUC5AC and MUC2 reduced the biofilm biomass by $67.6 \%$ and $75 \%$, respectively, compared to the mucin-free condition (Fig. 1e). Thus, both gastric and intestinal mucins disassemble $P$. aeruginosa biofilms. Due to its more facile purification, all subsequent experiments were performed with MUC5AC.

Mucins disassemble biofilms without affecting bacterial viability While the previous experiment showed that mucins can disintegrate biofilms (Fig. 1), a residual layer of attached cells always appeared to resist dissociation from the substrate. Hence, to determine whether cells that remained in the mucin-treated biofilm could be dissociated through longer exposure to mucins, we repeated the experiment and exposed $48 \mathrm{~h}$ PAO1-GFP biofilms to LB medium alone or to medium supplemented with $0.5 \%$ mucins, but this time extended the exposure time to $20 \mathrm{~h}$. After $20 \mathrm{~h}$, the mucin-treated biofilms remained lower in biomass (Fig. 2a) than biofilms not exposed to mucin (Fig. 2a), suggesting that mucins suppress biofilm development over a relatively long 

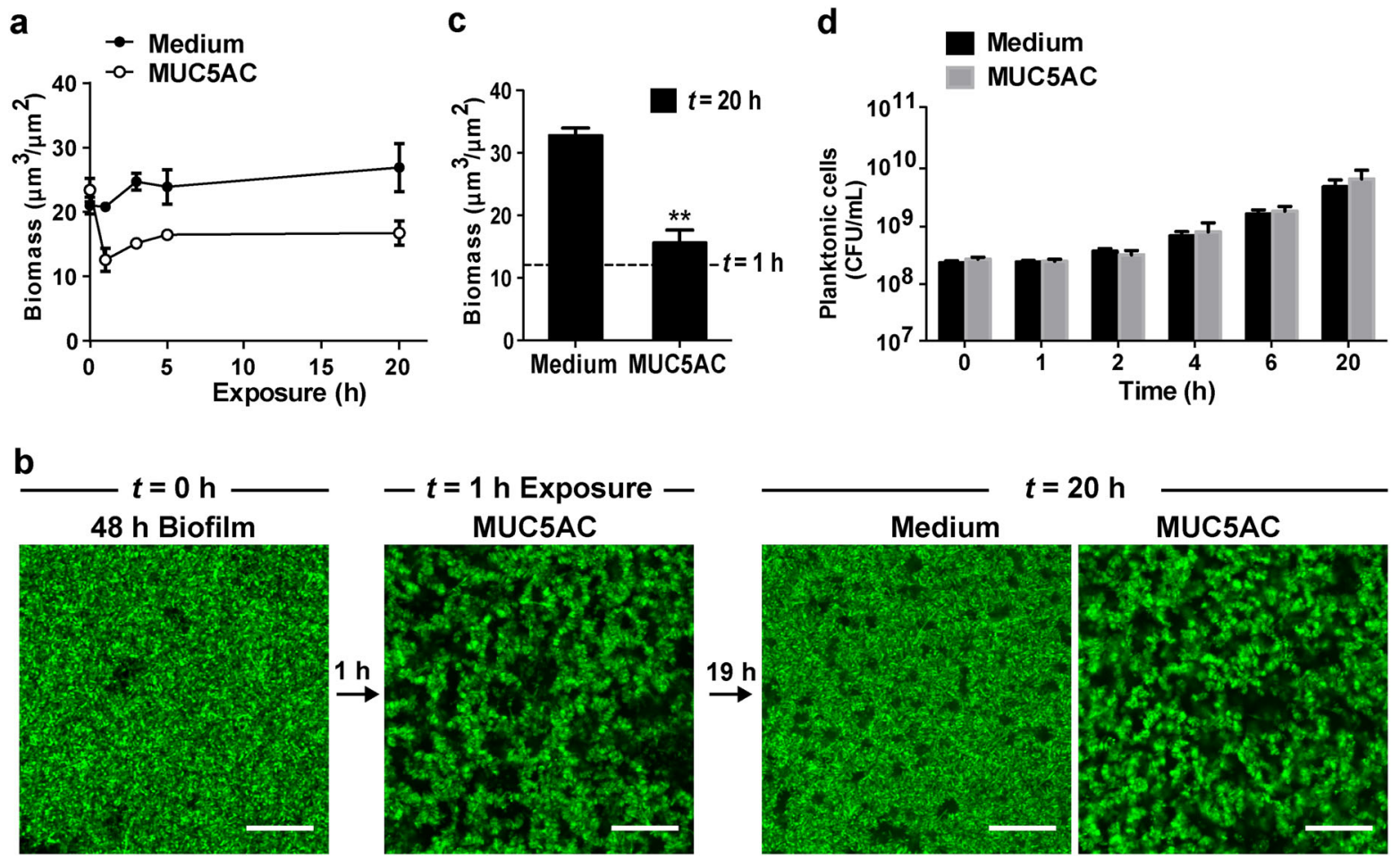

Fig. 2 Mucins disrupt $P$. aeruginosa biofilms without killing the bacteria. a Prolonged mucin exposure suppresses biofilm development. $48 \mathrm{~h}$ PAO1-GFP biofilms were exposed to medium with or without MUC5AC $(0.5 \% \mathrm{w} / \mathrm{v})$ over $20 \mathrm{~h}$ and the remaining surface-attached biofilm biomass quantified. Error bars represent standard error $(n=3)$. b Biofilms eroded by exposure to mucins remain viable. $48 \mathrm{~h}$ PAO1-GFP biofilms were first eroded via exposure to $0.5 \%$ MUC5AC for $1 \mathrm{~h}$ at $0.5 \mu \mathrm{L} / \mathrm{min}$ flow, then incubated for an additional $19 \mathrm{~h}$ in medium containing MUC5AC as indicated. Biofilms resumed development after being moved to mucin-free medium, indicating that eroded biofilms are still viable. Scale bars $=20 \mu \mathrm{m}$. c Quantification of biofilm biomass at $20 \mathrm{~h}$. Dotted lines indicate value after the initial $1 \mathrm{~h}$ mucin treatment. Error bars represent standard error $(n \geq 3)$. ${ }^{* *} P \leq 0.005$, unpaired Student's $t$ test. d Exposure to mucins does not significantly impair $P$. aeruginosa viability. PAO1-GFP cells were grown in suspension in medium with or without $0.5 \%$ mucins. Colony-forming units (CFUs) were counted to assess cell viability. Error bars represent standard error $(n=3)$

a

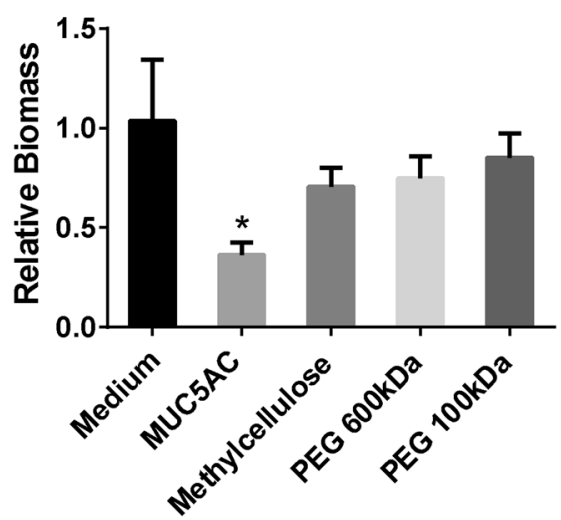

b

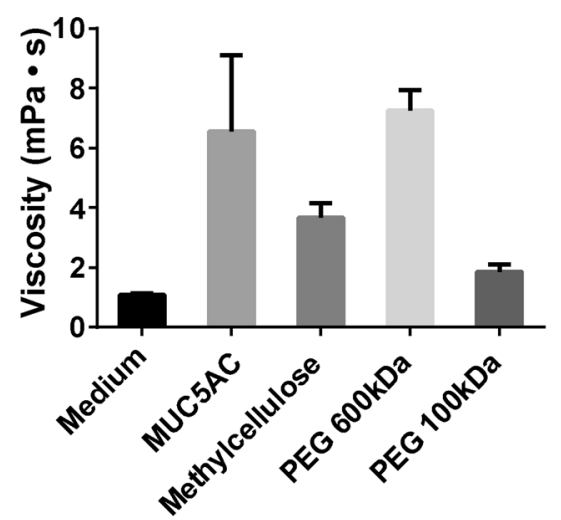

Fig. 3 Viscous polymer solutions do not disrupt $P$. aeruginosa biofilms to the same extent as mucins. a Quantification of PAO1-GFP biofilms biomass $(48 \mathrm{~h})$ after exposure to $0.5 \%(\mathrm{w} / \mathrm{v})$ polymer solutions in LB medium at $0.5 \mu \mathrm{L} / \mathrm{min}$ flow for $1 \mathrm{~h}$ shows that only mucins induce significant biofilm disassembly. Reported values represent biofilms after $1 \mathrm{~h}$ of exposure normalized to biofilms before exposure. Error bars represent standard error $(n=3)$. ${ }^{*} P \leq 0.05$ versus medium-only treatment, one-way ANOVA. The medium-only treatment and non-mucin polymer treatments did not significantly differ from each other. $\mathbf{b}$ Viscosity of polymers $(0.5 \%(\mathrm{w} / \mathrm{v})$ solutions, $n=3)$ is not associated with the ability to disrupt $P$. aeruginosa biofilms

period of time. However, the biofilms were not completely disintegrated, and a residual layer of biofilm cells remained (Fig. 2a).

We next determined whether this residual layer of cells could recover after mucin-mediated erosion. Biofilms were first treated with mucins for $1 \mathrm{~h}$ to trigger disassembly, then continually treated with mucins or allowed to recover through exposure to mucin-free medium for $19 \mathrm{~h}$ (Fig. 2b). Upon removal of the mucins, the biofilms began to resemble non-treated biofilms and increased in biomass nearly 3 -fold relative to the biomass at $1 \mathrm{~h}$ (Fig. 2b). These observations suggest that mucins dissociate the outer biofilm layers, leaving behind an eroded, but viable, biofilm 
a

\section{$48 \mathrm{~h}$ Biofilm}
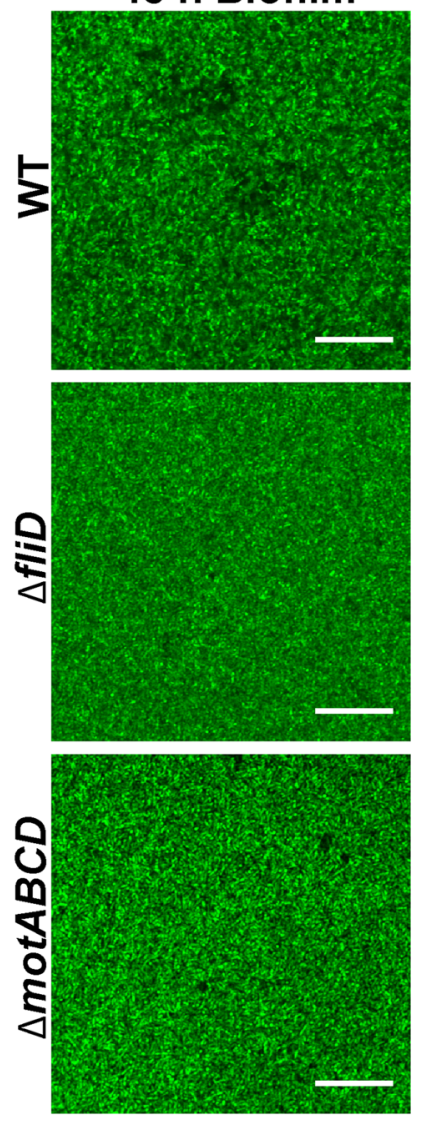

b

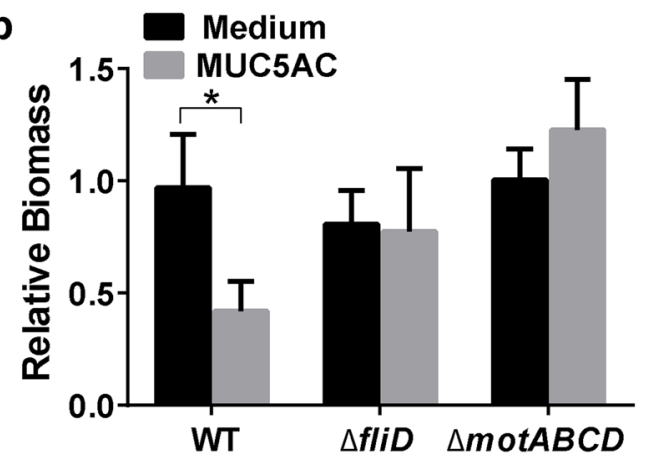

Fig. $4 P$. aeruginosa flagellar motility is required for mucinassociated biofilm erosion. a Live confocal imaging and $\mathbf{b}$ biofilm biomass quantification of PAO1-GFP (WT), PAO1 $\triangle$ fliD-GFP, and PAO1 $\triangle$ motABCD-GFP biofilms $(48 \mathrm{~h})$ exposed to LB medium with or without $0.5 \%$ mucins at $0.5 \mu \mathrm{L} / \mathrm{min}$ flow for $1 \mathrm{~h}$. Scale bars $=$ $20 \mu \mathrm{m}$. Biofilm biomass was quantified using COMSTAT analysis of confocal images. Reported values represent biofilms after $1 \mathrm{~h}$ of exposure normalized to WT biofilms before exposure $(t=0 \mathrm{~h})$. Error bars represent standard error $(n \geq 3)$. ${ }^{*} P \leq 0.05$, unpaired Student's $t$ test

that can regrow. In contrast, prolonged continuous exposure to mucins maintained a fragmented biofilm structure (Fig. 2b) and resulted in only a small increase of 1.4-fold in biofilm biomass (Fig. 2c). These data corroborate our observation that mucins hinder biofilm development (Fig. 2a).

Last, we addressed whether mucins disintegrate $P$. aeruginosa biofilms by killing the bacteria. We therefore evaluated the toxicity of mucins toward $P$. aeruginosa cells. Exposure to $0.5 \%$ MUC5AC over $20 \mathrm{~h}$ did not affect the growth rate of planktonic PAO1-GFP cells in static culture over this period (Fig. 2d), indicating that the viability of the planktonic cells was not reduced. The absence of bactericidal or bacteriostatic effects in planktonic cells suggests that mucins are generally non-toxic to the bacteria.

Biofilm disassembly is dependent on mucin-specific biochemistry To investigate the specificity of mucin-mediated biofilm disassembly, we assessed the ability of several commonly studied synthetic polymer solutions to disrupt biofilms. Since mucins are heavily glycosylated polymers, ${ }^{14,33}$ we compared them to glycanbased polymers like methylcellulose, which has been studied as a mucin mimetic due to its similar viscoelastic properties, ${ }^{34,35}$ and non-glycan polymers like polyethylene glycol (PEG), which is commonly used in antifouling coatings. ${ }^{36}$ PAO1-GFP biofilms exposed to $0.5 \%$ methylcellulose or $0.5 \%$ PEG (600 or $100 \mathrm{kDa}$ ) for $1 \mathrm{~h}$ did not exhibit substantial biofilm disassembly (Fig. 3a). Methylcellulose treatment caused a $29.4 \%$ reduction in biomass, and 600 and $100 \mathrm{kDa}$ PEG treatments resulted in similar outcomes (Fig. 3a). These data indicate that while biofilms are disrupted by other polymers, mucins exert a greater effect.

Because the viscosity of polymer solutions contributes to biofilm-disrupting shear stress, ${ }^{30-32}$ the rheological properties of the $0.5 \%$ polymer solutions were characterized. Mucin and $600 \mathrm{kDa}$ PEG solutions had the highest viscosities, followed by methylcellulose and $100 \mathrm{kDa}$ PEG (Fig. 3b). Thus, since the mucins and the $600 \mathrm{kDa}$ PEG solutions had similar viscosities, but mucins more effectively disassembled biofilms, the viscosity of the polymer solutions is not directly associated with the extent of biofilm disruption. Since neither the glycan-based nor non-glycan synthetic polymers tested here disassembled the biofilms to the same degree as mucins (despite similarities in viscosity; Fig. 3b), unique biochemical or molecular properties of mucins may underlie the disruption of $P$. aeruginosa biofilms.

Mucin-mediated biofilm disruption is dependent on the $P$. aeruginosa flagellum

Previous studies have shown that flagella, the motility appendages required for bacterial swimming through the liquid, play an important role in $P$. aeruginosa interactions with mucins. ${ }^{37-39}$ In addition, flagella are involved in bacterial dispersal from biofilms ${ }^{40,41}$ and thus may contribute to mucin-associated biofilm disruption. We therefore studied biofilms of $P$. aeruginosa lacking the flagellar cap FliD or flagellar stators MotABCD; these mutants displayed impaired swimming motility (Figure S1). When PAO1 $\triangle$ fliD-GFP biofilms were grown in flow cells for $48 \mathrm{~h}$, they closely resembled $48 \mathrm{~h}$ PAO1 wild-type (WT) biofilms (Fig. 4a). In contrast to WT biofilms, $\Delta$ fliD biofilms did not exhibit a substantial reduction in biomass after treatment with $0.5 \%$ mucins (Fig. $4 \mathrm{~b}$ ). The resistance of fliD mutant biofilms to mucin-mediated disruption suggests the involvement of FliD in the phenomenon.

We next examined biofilms of swimming-impaired $\triangle \operatorname{mot} A B C D$ bacteria, which produce intact, but paralyzed, flagella. ${ }^{42}$ PAO1 $\triangle$ motABCD-GFP biofilms grown for $48 \mathrm{~h}$ in flow cells resembled WT biofilms (Fig. 4a). Similar to the $\triangle$ fliD biofilms, $\triangle$ mot $A B C D$ biofilms treated with $0.5 \%$ MUC5AC for $1 \mathrm{~h}$ did not disassemble (Fig. $4 \mathrm{a}, \mathrm{b}$ ). Thus, flagellar motility is involved in the biofilm response to mucins. Mucins may stimulate active biofilm dispersal, which in $P$. aeruginosa involves swimming motility and thus functional flagella. ${ }^{40,41}$

Mucins trigger active cellular escape from biofilms

If mucins induce a biofilm-escape mechanism that relies on flagellar motility, then mucin-mediated biofilm disintegration should also occur in the absence of fluid flow. To test this hypothesis, we cultured biofilms in static conditions for $48 \mathrm{~h}$ in 
a

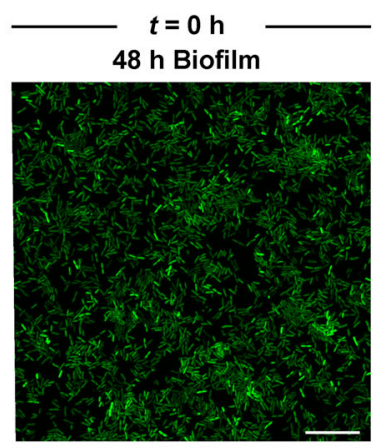

b
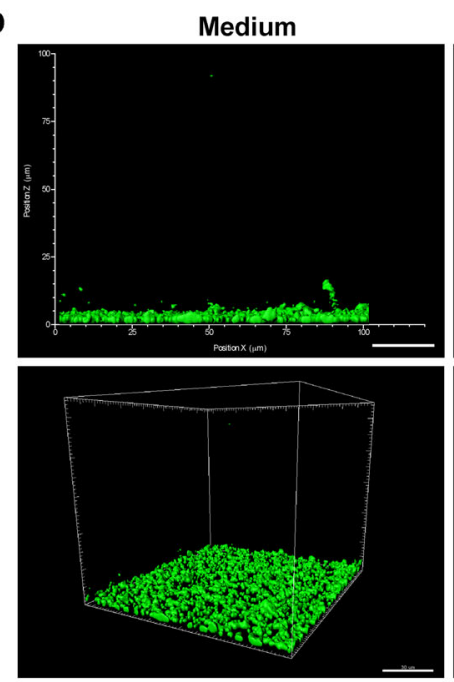
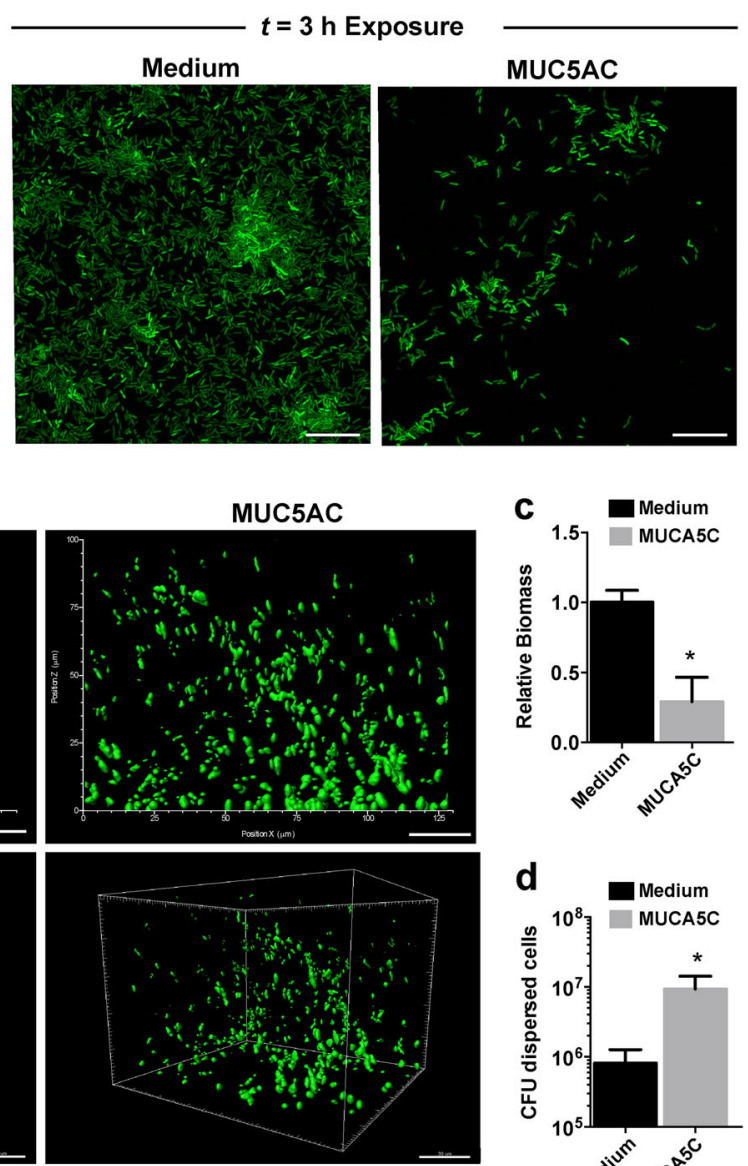

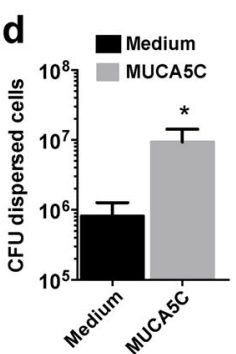

Fig. 5 Exposure to mucins induces dispersal of $P$. aeruginosa biofilms under static growth conditions. a Mucins prompt active biofilm dispersal in P. aeruginosa. Live confocal imaging of $48 \mathrm{~h}$ PAO1-GFP biofilms before $(t=0 \mathrm{~h})$ and after $(t=3 \mathrm{~h})$ exposure to ABTG minimal medium or ABTG medium $+0.5 \%(\mathrm{w} / \mathrm{v})$ MUC5AC. Scale bars $=20 \mu \mathrm{m}$. b Dispersed cells display a distinctive spatial organization. 3D representation of the cellular distribution of dispersed $48 \mathrm{~h}$ PAO1-GFP biofilms after $3 \mathrm{~h}$ of exposure to ABTG medium or to ABTG medium $+0.5 \%$ mucins. c Exposure to mucins reduces biomass of $P$. aeruginosa biofilms. Values are normalized to biofilm biomass before exposure. Error bars represent standard error $(n \geq 3)$. ${ }^{*} P \leq 0.05$, unpaired Student's $t$ test. d Exposure to mucins increases the number of dispersed cells from $P$. aeruginosa biofilms. Viable dispersed cells were quantified by counting colony-forming units (CFUs). Error bars represent standard error ( $n \geq 3$ )

glass-bottom 96-well plates and exposed them to medium containing $0.5 \%$ MUC5AC for $3 \mathrm{~h}$. Confocal imaging revealed that biofilms exposed to mucin-free medium remained unaltered (Fig. $5 \mathrm{a}, \mathrm{c})$. In contrast, exposure to mucins reduced the surfaceattached biofilm biomass by $70.8 \%$ (Fig. $5 \mathrm{a}$, c). At the same time, after exposure to mucins, cellular aggregates of bacteria had lifted off the surface and were detected in the volume of the medium above the biofilm (Fig. 5b). These dispersed aggregates contained viable bacteria and contained a 10-fold higher cell count than the medium above untreated biofilms (Fig. 5d). This mucin-mediated disintegration of biofilms was not observed with non-motile $\Delta$ fliD cells (Figure S2). Although natively purified mucins are obtained through a relatively mild purification procedure to preserve their structure, it is possible that mucin-associated factors contribute to biofilm disintegration. To exclude this possibility, we tested mucins purified with cesium chloride, which removes the majority of associated proteins and lipids. ${ }^{43,44}$ Cesium chloride-purified mucins reduced $P$. aeruginosa biofilm biomass to a similar degree as natively purified mucins (Figure S3), indicating that this effect is likely due to the mucins and not to associated factors. These data suggest that mucins trigger an active bacterial biofilm escape, a key characteristic of biofilm dispersal. ${ }^{41,45,46}$

\section{DISCUSSION}

The mucosal barrier plays a critical role in maintaining homeostasis with host-associated biofilms. Here, we report that mucin polymers induce the disassembly of $P$. aeruginosa biofilms. Specifically, exposure to mucins resulted in disassembly of the outer layers of the biofilm, leaving behind a viable, yet eroded, structure. Mucin-induced biofilm dispersion depended on the flagellar cap encoded by fliD and flagellar stators encoded by $\operatorname{mot} A B C D$, suggesting that flagellar motility is one component of the disassembly mechanism.

There are several mechanisms by which mucins may act upon $P$. aeruginosa biofilms. First, mucins could directly or indirectly influence bacterial signaling. For example, $P$. aeruginosa directly responds to $\mathrm{N}$-acetylglucosamine, a glycan found on mucins, via a two-component regulator. ${ }^{47}$ Mucins may also trigger signaling events indirectly by altering nutrient availability and environmental conditions. ${ }^{41,46,48}$ Moreover, mucin-associated glycans may interact with specific $P$. aeruginosa surface adhesins involved in biofilm dispersion. ${ }^{49}$ Last, heavily glycosylated host mucins may compete against native $P$. aeruginosa polysaccharides for binding sites on the bacterial surface and within the biofilm matrix, thereby disrupting primary interactions that underlie biofilm 
development. A growing body of evidence points to the disruptive nature of foreign polysaccharides on the development and organization of bacterial biofilms. ${ }^{50-53}$

Here, we identified the flagellum as a key factor in the response of $P$. aeruginosa biofilms to mucins, consistent with previous reports that flagellar structural proteins in $P$. aeruginosa interact directly with mucins. ${ }^{39,54,55}$ It is possible that upon binding to mucins, the flagellum triggers intracellular signaling pathways. The importance of flagellar motility in mucin-induced biofilm disintegration is also consistent with our previous observation that mucins suppress the aggregation of $P$. aeruginosa cells into biofilm-like flocs by retaining them in their free-swimming planktonic state. ${ }^{22}$ Both the suppression of cellular aggregation and the disassembly of established biofilms observed here require active flagellar motility and are likely mediated by similar signaling pathways.

While exposure to mucins substantially eroded biofilms in both flow and static systems, complete biofilm eradication was not achieved. Biofilm regions that remained intact after mucin treatment may differ physiologically ${ }^{56,57}$ from those that are eroded; alternatively, subpopulations within the biofilm may vary in their ability to sense or respond to mucins.

Our work highlights the potential of mucins, at physiological concentrations, $^{58-60}$ to disrupt biofilms of the problematic opportunistic pathogen $P$. aeruginosa, and points to the importance of including these polymers in experimental models of mucosal microbial pathogenesis. Can mucins impact biofilms formed by other microorganisms, or sensitize biofilms to certain antibiotics? A better understanding of the extent and mechanisms of mucin-biofilm interactions may elucidate how mucus impacts bacterial behavior in mucosal environments in vivo. From a therapeutic perspective, the current work indicates that native purified mucins may be leveraged to improve the efficacy of biofilm-eradicating treatments. One important challenge now is to understand the specific changes in abnormal mucins and mucus that allow biofilm formation in chronic diseases such as cystic fibrosis. ${ }^{14,15}$ Matsui et al. ${ }^{61}$ reported that both cystic fibrosis mucus and dehydrated healthy mucus, but not fully hydrated healthy mucus, promote the formation of dense colonies of $P$. aeruginosa. While the dehydrated mucus of the cystic-fibrosis lung appears to support biofilm formation of $P$. aeruginosa, the specific attributes distinguishing diseased mucus from healthy mucus have yet to be elucidated. Systematic studies comparing mucus in health and disease will be fundamental to identifying specific properties and components of mucus and mucins that confer anti-biofilm effects. Such work will provide a foundation for novel mucin-inspired strategies aimed at preventing biofilm-related disease and restoring diseased mucus to a healthy state.

\section{MATERIALS AND METHODS}

\section{Ethics statement}

The use of porcine tissues for mucin purification in this study was approved by the MIT IACUC Committee on Animal Care (CAC protocol number E13-07-0416). All protocols conformed to the USDA Animal Welfare Act and the NIH Public Health Service Policy on Humane Care and Use of Laboratory Animals.

\section{Bacterial strains and culture conditions}

$P$. aeruginosa WT strain PAO1 and its derivatives PAO1 $\triangle$ fliD and PAO1 $\triangle$ motABCD have been previously described. ${ }^{22}$ For constitutive GFP expression, strains were transformed with pBBR1(MCS5)-Plac-gfp. ${ }^{62}$ Standard electroporation methods were employed to produce PAO1-GFP, PAO1 $\triangle$ fliD-GFP, and PAO1 $\triangle$ motABCD-GFP strains.

$P$. aeruginosa strains were grown in LB (Difco) with $30 \mu \mathrm{g} / \mathrm{mL}$ gentamicin to maintain the plasmid. For determination of planktonic cell viability, bacterial cultures were serially diluted in phosphate-buffered saline and plated on LB agar for CFU counts. $\mathrm{OD}_{600}$ of 0.0025 represents a culture density of $\sim 5.0 \times 10^{5} \mathrm{CFU} / \mathrm{mL}$.

\section{Mucin purification}

This study used native porcine gastric mucins, which differ from industrially purified mucins in their rheological properties. ${ }^{27}$ Native mucins were purified as previously reported. ${ }^{63}$ Briefly, mucus was scraped from fresh pig stomachs (Research 87, Inc., Boylston, MA, USA) and solubilized in $\mathrm{NaCl}$ buffer with protease inhibitors and sodium azide. Insoluble material was pelleted via ultracentrifugation and mucins were purified using sizeexclusion chromatography on a Sepharose CL-2B column (GE Healthcare Life Sciences). Mucin fractions were desalted, concentrated, and lyophilized for storage. Intestinal mucins were isolated from the mucus of fresh porcine small intestines and purified as described above for native gastric mucins. To ensure that there were no contaminants in the mucin preparation, mucins prepared via $\mathrm{CsCl}$ gradient centrifugation as previously reported ${ }^{43,44}$ were compared to those prepared without this step.

\section{Flow-cell biofilm reactor}

Biofilms were grown in flow cells supplied with LB medium. The flow system was assembled and prepared as described previously. ${ }^{62}$ Briefly, a polydimethylsiloxane (Sylgard 184; Dow Corning) microfluidic device was molded from capillaries anchored to a petri dish, yielding a negative imprint of four straight microchannels $(4 \times 2 \times 35 \mathrm{~mm})$; this imprint was bonded to a glass coverslip. A suspension of cells at $\mathrm{OD}_{600}=0.0025$ was introduced into the microchannels under continuous flow $(0.5 \mu \mathrm{L} / \mathrm{min}$ unless indicated otherwise) driven by a syringe pump (PHD Ultra, Harvard Apparatus, Holliston MA, USA) for $48 \mathrm{~h}$ at room temperature. Fresh medium was introduced for $3 \mathrm{~min}$ at $25 \mu \mathrm{L} / \mathrm{min}$ before treatment or analysis to remove the unattached cells from the biofilm surface, allowing for clearer detection of the attached cells by confocal microscopy. This wash step is included in control treatments (mucin-free media) and does not affect biofilm morphology. Mucins, methylcellulose (15 CP SigmaAldrich), PEG 100 kDa (Sigma-Aldrich), or PEG 600 kDa (Sigma-Aldrich) in LB were introduced into channels at $0.5 \%(\mathrm{w} / \mathrm{v})$, unless otherwise stated, for the indicated treatment times. Biofilms were imaged before and after treatments at the middle of the channel using a Zeiss 510 confocal laserscanning microscope with a $20 \times / 0.5$ NA dry objective with $2 \times$ or $4 \times$ zoom or a $100 \times / 1.4$ NA oil immersion objective for high-magnification images. The excitation wavelength for GFP was $488 \mathrm{~nm}$. One stack was recorded for each flow cell and at least three independent flow cells were analyzed for each condition. Images were acquired with a step size of $0.5 \mu \mathrm{m}$. Fluorescence signal from GFP-expressing bacteria was quantified using COMSTAT $^{64}$ to determine biofilm biomass. Biofilm biomass after treatments is reported as absolute values $\left(\mu \mathrm{m}^{3} / \mu \mathrm{m}^{2}\right)$ or as relative biomass (normalized to biofilm biomass before treatments).

\section{Rheology}

Rheological tests were performed on an MCR 302 rheometer (Anton-Paar) in a cone-plate geometry. The diameter of the cone was $25 \mathrm{~mm}$, the cone angle was $1^{\circ}$, and the cone truncation was $51 \mu \mathrm{m}$. Ninety microliters of $0.5 \%$ polymer solutions solubilized in LB were applied to the rheometer. Shear stress was measured at shear rates of $\dot{\gamma}=10 / \mathrm{s}$ to $100 / \mathrm{s}$. Viscosity was calculated assuming a Newtonian relationship between the stress and the shear rate, $\tau(\dot{\gamma})=\eta \dot{\gamma}$.

\section{Biofilm dispersal under static conditions}

$P$. aeruginosa biofilm dispersal was assayed as previously described ${ }^{65}$ with the following modifications. Briefly, an overnight culture of PAO1-GFP was prepared in LB with shaking at $37^{\circ} \mathrm{C}$. Overnight cultures were diluted in ABTG medium to an initial $\mathrm{OD}_{600}$ of 0.01 , added to a glass-bottom 96 -well plate, and incubated for $48 \mathrm{~h}$ at $37^{\circ} \mathrm{C}$. The supernatant containing nonadherent cells was removed from the plate and the biofilm remaining in each well was washed at least three times with $0.9 \% \mathrm{NaCl}$. Biofilms were exposed to ABTG medium alone or $\mathrm{ABTG}$ medium $+0.5 \%$ mucins (MUC5AC) and incubated at $37^{\circ} \mathrm{C}$ with gentle shaking $(<80 \mathrm{rpm})$ for $3 \mathrm{~h}$. Plates were either examined via microscopy to determine the cellular distribution of dispersed cells in each well or the biofilms in all wells were washed three times with $0.9 \% \mathrm{NaCl}$ and resuspended in ABTG medium for microscopy to quantify the remaining biofilm biomass. Viable dispersed 
cells were quantified via CFU counts on LB agar plates. Experiments were performed in triplicate.

Image acquisition was performed using a confocal laser scanning microscope (LSM 800; Zeiss) equipped with a $63 \times / 1.4$ NA oil immersion or a $100 \times / 1.4$ NA oil immersion objective. Images were analyzed with Zeiss ZEN 2.1 imaging software (Thornwood, NY, USA). The excitation wavelength for GFP was $488 \mathrm{~nm}$. At least five stacks were recorded for each well and at least three independent wells were analyzed for each condition. Images were acquired with a step size of $0.5 \mu \mathrm{m}$. Biofilm quantification was performed using COMSTAT $1 .{ }^{66} 3 \mathrm{D}$ images of biofilms and planktonic cells (Fig. 5b) were created with IMARIS 7.7.2 (Bitplane, Switzerland).

\section{Swimming motility}

Swimming motility was evaluated using a previously described plate-based assay. ${ }^{67}$ P. aeruginosa strains were grown overnight on LB agar plates at $37^{\circ} \mathrm{C}$. Using a sterile pipette tip, colonies were picked and stabbed into swimming agar plates (M8 salts, $1 \mathrm{mM} \mathrm{MgSO}_{4}, 0.2 \%$ glucose, $0.5 \%$ casamino acids, $0.3 \%$ agar). Plates were incubated upright for $24 \mathrm{~h}$ at room temperature, and diameters of the swim zones were measured.

\section{Statistical analysis}

Analyses were performed using PRISM (GraphPad Software). To determine the statistical significance of the difference between the means for two experimental groups, an unpaired, two-tailed Student's $t$ test was used. For the polymer-treatment experiment in which multiple experimental conditions were compared to the LB control, one-way ANOVA with Dunnett's multiple comparison test was used. Differences were considered statistically significant if $P \leq 0.05$.

\section{DATA AVAILABILITY}

The authors declare that all relevant data supporting the findings of the study are available in this article and its Supplementary Information files, or from the corresponding author upon request.

\section{ACKNOWLEDGEMENTS}

This work was supported by NIBIB/NIH Grant R01 EB017755-02, Charles E. Reed Faculty Initiative Funds (to K.R.), a Preterm Birth Research Grant from the Burroughs Wellcome Fund (to K.R.), and NIEHS/NIH Grant P30-ES002109. N.B. was supported by NIEHS/NIH Training Grant in Toxicology T32 ES7020-37. S.C.G. and N.H.-A. were supported by the MRSEC program of the National Science Foundation under award DMR 1419807. G.C. was supported by the Early Postdoc Mobility Fellowship of the Swiss National Science Foundation (grant P2ZHP3_164844). K.W. is supported by the MIT/NIGMS Biotechnology Training Program (grant 5T32GM008334-28). The funders had no role in study design, data collection and interpretation, or the decision to submit this work for publication. We thank Nicole Kavanaugh for helpful comments on the manuscript, and Brad Turner for providing $\mathrm{CsCl}$-purified mucins.

\section{AUTHOR CONTRIBUTIONS}

J.C., G.C.-O., K.W., N.B., and K.R. designed the experiments, J.C., G.C.-O., K.W., and S.G. conducted experiments. All authors analyzed the data and contributed to the writing of the manuscript.

\section{ADDITIONAL INFORMATION}

Supplementary information accompanies the paper on the npj Biofilms and Microbiomes website (https://doi.org/10.1038/s41522-018-0067-0).

Competing interests: The authors declare no competing interests.

Publisher's note: Springer Nature remains neutral with regard to jurisdictional claims in published maps and institutional affiliations.

\section{REFERENCES}

1. O'Toole, G., Kaplan, H. B. \& Kolter, R. Biofilm formation as microbial development. Annu. Rev. Microbiol. 54, 49-79 (2000).
2. Hall-Stoodley, L., Costerton, J. W. \& Stoodley, P. Bacterial biofilms: from the natural environment to infectious diseases. Nat. Rev. Microbiol. 2, 95-108 (2004).

3. Flemming, H. C., Neu, T. R. \& Wozniak, D. J. The EPS matrix: the 'house of biofilm cells'. J. Bacteriol. 189, 7945-7947 (2007).

4. Darouiche, R. O. Device-associated infections: a macroproblem that starts with microadherence. Clin. Infect. Dis. 33, 1567-1572 (2001).

5. Wozniak, D. J. et al. Alginate is not a significant component of the extracellular polysaccharide matrix of PA14 and PAO1 Pseudomonas aeruginosa biofilms. Proc. Natl. Acad. Sci. U.S.A. 100, 7907-7912 (2003).

6. Hall-Stoodley, L. \& Stoodley, P. Biofilm formation and dispersal and the transmission of human pathogens. Trends Microbiol. 13, 7-10 (2005).

7. Magill, S. S. et al. Multistate point-prevalence survey of health care-associated infections. N. Engl. J. Med. 370, 1198-1208 (2014).

8. Johansson, M. E. et al. The inner of the two Muc2 mucin-dependent mucus layers in colon is devoid of bacteria. Proc. Natl. Acad. Sci. U.S.A. 105, 15064-15069 (2008).

9. Linden, S. K., Sutton, P., Karlsson, N. G., Korolik, V. \& McGuckin, M. A. Mucins in the mucosal barrier to infection. Mucosal Immunol. 1, 183-197 (2008).

10. Derrien, M. et al. Mucin-bacterial interactions in the human oral cavity and digestive tract. Gut Microbes 1, 254-268 (2010).

11. McGuckin, M. A., Lindén, S. K., Sutton, P. \& Florin, T. H. Mucin dynamics and enteric pathogens. Nat. Rev. Microbiol. 9, 265-278 (2011).

12. Bansil, R. \& Turner, B. S. Mucin structure, aggregation, physiological functions and biomedical applications. Curr. Opin. Colloid Interface Sci. 11, 164-170 (2006).

13. Hattrup, C. L. \& Gendler, S. J. Structure and function of the cell surface (tethered) mucins. Annu. Rev. Physiol. 70, 431-457 (2008).

14. Rose, M. C. \& Voynow, J. A. Respiratory tract mucin genes and mucin glycoproteins in health and disease. Physiol. Rev. 86, 245-278 (2006).

15. Henke, M. O., John, G., Germann, M., Lindemann, H. \& Rubin, B. K. MUC5AC and MUC5B mucins increase in cystic fibrosis airway secretions during pulmonary exacerbation. Am. J. Respir. Crit. Care Med. 175, 816-821 (2007).

16. Caramori, G. et al. Mucin expression in peripheral airways of patients with chronic obstructive pulmonary disease. Histopathology 45, 477-484 (2004).

17. Van der Sluis, M. et al. Muc2-deficient mice spontaneously develop colitis, indicating that MUC2 is critical for colonic protection. Gastroenterology 131, 117-129 (2006).

18. Heazlewood, C. K. et al. Aberrant mucin assembly in mice causes endoplasmic reticulum stress and spontaneous inflammation resembling ulcerative colitis. PLoS Med. 5, e54 (2008).

19. Tilley, A. E., Walters, M. S., Shaykhiev, R. \& Crystal, R. G. Cilia dysfunction in lung disease. Annu. Rev. Physiol. 77, 379-406 (2015).

20. Zemanick, E. T. \& Hoffman, L. R. Cystic fibrosis: microbiology and host response. Pediatr. Clin. North Am. 63, 617-636 (2016).

21. Kavanaugh, N. L., Zhang, A. Q., Nobile, C. J., Johnson, A. D. \& Ribbeck, K. Mucins suppress virulence traits of Candida albicans. mBio 5, e01911 (2014).

22. Caldara, M. et al. Mucin biopolymers prevent bacterial aggregation by retaining cells in the free-swimming state. Curr. Biol. 22, 2325-2330 (2012).

23. Celli, J. P. et al. Helicobacter pylori moves through mucus by reducing mucin viscoelasticity. Proc. Natl. Acad. Sci. U.S.A. 106, 14321-14326 (2009).

24. Stewart, P. S. \& Costerton, J. W. Antibiotic resistance of bacteria in biofilms. Lancet 358, 135-138 (2001)

25. Høiby, N., Bjarnsholt, T., Givskov, M., Molin, S. \& Ciofu, O. Antibiotic resistance of bacterial biofilms. Int. J. Antimicrob. Agents 35, 322-332 (2010).

26. Bjarnsholt, T. et al. The in vivo biofilm. Trends Microbiol. 21, 466-474 (2013).

27. Kocevar-Nared, J., Kristl, J. \& Smid-Korbar, J. Comparative rheological investigation of crude gastric mucin and natural gastric mucus. Biomaterials 18, 677-681 (1997).

28. Crater, J. S. \& Carrier, R. L. Barrier properties of gastrointestinal mucus to nanoparticle transport. Macromol. Biosci. 10, 1473-1483 (2010).

29. Billings, N., Rusconi, R., Stocker, R. \& Ribbeck, K. Microfluidic-based time-kill kinetic assay. Bio-Protocol 4, e1116 (2014).

30. Wanner, O., Cunningham, A. B. \& Lundman, R. Modeling biofilm accumulation and mass transport in a porous medium under high substrate loading. Biotechnol. Bioeng. 47, 703-712 (1995).

31. Stoodley, P., Lewandowski, Z., Boyle, J. D. \& Lappin-Scott, H. M. Structural deformation of bacterial biofilms caused by short-term fluctuations in fluid shear: an in situ investigation of biofilm rheology. Biotechnol. Bioeng. 65, 83-92 (1999).

32. Stoodley, P. et al. Growth and detachment of cell clusters from mature mixedspecies biofilms. Appl. Environ. Microbiol. 67, 5608-5613 (2001).

33. Thornton, D. J. \& Sheehan, J. K. From mucins to mucus: toward a more coherent understanding of this essential barrier. Proc. Am. Thorac. Soc. 1, 54-61 (2004).

34. Worku, M. L. et al. Motility of Helicobacter pylori in a viscous environment. Eur. J. Gastroenterol. Hepatol. 11, 1143-1150 (1999). 
35. Smith, D. J., Gaffney, E. A., Gadelha, H., Kapur, N. \& Kirkman-Brown, J. C. Bend propagation in the flagella of migrating human sperm, and its modulation by viscosity. Cell Motil. Cytoskelet. 66, 220-236 (2009).

36. Banerjee, I., Pangule, R. C. \& Kane, R. S. Antifouling coatings: recent developments in the design of surfaces that prevent fouling by proteins, bacteria, and marine organisms. Adv. Mater. 23, 690-718 (2011).

37. Arora, S. K., Ritchings, B. W., Almira, E. C., Lory, S. \& Ramphal, R. The Pseudomonas aeruginosa flagellar cap protein, FliD, is responsible for mucin adhesion. Infect. Immun. 66, 1000-1007 (1998).

38. Scharfman, A. et al. Recognition of Lewis $x$ derivatives present on mucins by flagellar components of Pseudomonas aeruginosa. Infect. Immun. 69, 5243-5248 (2001).

39. Landry, R. M., An, D., Hupp, J. T., Singh, P. K. \& Parsek, M. R. Mucin-Pseudomonas aeruginosa interactions promote biofilm formation and antibiotic resistance. Mol. Microbiol. 59, 142-151 (2006).

40. Sauer, K., Camper, A. K., Ehrlich, G. D., Costerton, J. W. \& Davies, D. G. Pseudomonas aeruginosa displays multiple phenotypes during development as a biofilm. J. Bacteriol. 184, 1140-1154 (2002).

41. Davies, D. G. Biofilm dispersion. In Biofilm Highlights (eds. Flemming, H.-C., Wingender, J. \& Szewzyk, U.) Vol. 5, 1-28 (Springer, Berlin, Heidelberg, 2011).

42. Toutain, C. M., Zegans, M. E. \& O'Toole, G. A. Evidence for two flagellar stators and their role in the motility of Pseudomonas aeruginosa. J. Bacteriol. 187, 771-777 (2005).

43. Smith, B. F. \& LaMont, J. T. Hydrophobic binding properties of bovine gallbladder mucin. J. Biol. Chem. 259, 12170-12177 (1984).

44. Gong, D. H., Turner, B., Bhaskar, K. R. \& Lamont, J. T. Lipid binding to gastric mucin: protective effect against oxygen radicals. Am. J. Physiol. 259, G681-G686 (1990).

45. Chua, S. L. et al. Dispersed cells represent a distinct stage in the transition from bacterial biofilm to planktonic lifestyles. Nat. Commun. 5, 4462 (2014).

46. Petrova, O. E. \& Sauer, K. Escaping the biofilm in more than one way: desorption, detachment or dispersion. Curr. Opin. Microbiol. 30, 67-78 (2016).

47. Korgaonkar, A., Trivedi, U., Rumbaugh, K. P. \& Whiteley, M. Community surveillance enhances Pseudomonas aeruginosa virulence during polymicrobial infection. Proc. Natl. Acad. Sci. U.S.A. 110, 1059-1064 (2013).

48. McDougald, D., Rice, S. A., Barraud, N., Steinberg, P. D. \& Kjelleberg, S. Should we stay or should we go: mechanisms and ecological consequences for biofilm dispersal. Nat. Rev. Microbiol. 10, 39-50 (2011).

49. Johansson, E. M. et al. Inhibition and dispersion of Pseudomonas aeruginosa biofilms by glycopeptide dendrimers targeting the fucose-specific lectin LecB. Chem. Biol. 15, 1249-1257 (2008).

50. Karwacki, M. T. et al. Antibiofilm activity of Actinobacillus pleuropneumoniae serotype 5 capsular polysaccharide. PLoS One 8, e63844 (2013).

51. Rendueles, O., Kaplan, J. B. \& Ghigo, J. M. Antibiofilm polysaccharides. Environ. Microbiol. 15, 334-346 (2013).

52. Murugan, K., Selvanayaki, K. \& Al-Sohaibani, S. Urinary catheter indwelling clinical pathogen biofilm formation, exopolysaccharide characterization and their growth influencing parameters. Saudi J. Biol. Sci. 23, 150-159 (2016).

53. Wu, S., Liu, G., Jin, W., Xiu, P. \& Sun, C. Antibiofilm and anti-Infection of a marine bacterial exopolysaccharide against Pseudomonas aeruginosa. Front. Microbiol. 7, 102 (2016).
54. Simpson, D. A., Ramphal, R. \& Lory, S. Genetic analysis of Pseudomonas aeruginosa adherence: distinct genetic loci control attachment to epithelial cells and mucins. Infect. Immun. 60, 3771-3779 (1992).

55. Arora, S. K., Ritchings, B. W., Almira, E. C., Lory, S. \& Ramphal, R. Cloning and characterization of Pseudomonas aeruginosa fliF, necessary for flagellar assembly and bacterial adherence to mucin. Infect. Immun. 64, 2130-2136 (1996).

56. Tolker-Nielsen, T. \& Molin, S. Spatial organization of microbial biofilm communities. Microb. Ecol. 40, 75-84 (2000).

57. Stewart, P. S. \& Franklin, M. J. Physiological heterogeneity in biofilms. Nat. Rev. Microbiol. 6, 199-210 (2008).

58. Fahy, J. V. \& Dickey, B. F. Airway mucus function and dysfunction. N. Engl. J. Med. 363, 2233-2247 (2010).

59. Adler, K. B., Tuvim, M. J. \& Dickey, B. F. Regulated mucin secretion from airway epithelial cells. Front. Endocrinol. 4, 129 (2013).

60. Livraghi-Butrico, A. et al. Contribution of mucus concentration and secreted mucins Muc5ac and Muc5b to the pathogenesis of muco-obstructive lung disease. Mucosal Immunol. 10, 395-407 (2017).

61. Matsui, $H$. et al. A physical linkage between cystic fibrosis airway surface dehydration and Pseudomonas aeruginosa biofilms. Proc. Natl. Acad. Sci. U.S.A. 103, 18131-18136 (2006)

62. Billings, N. et al. The extracellular matrix component Psl provides fast-acting antibiotic defense in Pseudomonas aeruginosa biofilms. PLoS Pathog. 9, e1003526 (2013).

63. Lieleg, O., Lieleg, C., Bloom, J., Buck, C. B. \& Ribbeck, K. Mucin biopolymers as broad-spectrum antiviral agents. Biomacromolecules 13, 1724-1732 (2012).

64. Heydorn, A. et al. Quantification of biofilm structures by the novel computer program COMSTAT. Microbiology 146(Pt 1), 2395-2407 (2000).

65. Chua, S. L. et al. In vitro and in vivo generation and characterization of Pseudomonas aeruginosa biofilm-dispersed cells via c-di-GMP manipulation. Nat. Protoc. 10, 1165-1180 (2015)

66. Heydorn, A. et al. Experimental reproducibility in flow-chamber biofilms. Microbiology 146(Pt 1), 2409-2415 (2000).

67. Ha, D. G., Kuchma, S. L. \& O’Toole, G. A. Plate-based assay for swimming motility in Pseudomonas aeruginosa in. Methods Mol. Biol. 1149, 59-65 (2014).

Open Access This article is licensed under a Creative Commons Attribution 4.0 International License, which permits use, sharing, adaptation, distribution and reproduction in any medium or format, as long as you give appropriate credit to the original author(s) and the source, provide a link to the Creative Commons license, and indicate if changes were made. The images or other third party material in this article are included in the article's Creative Commons license, unless indicated otherwise in a credit line to the material. If material is not included in the article's Creative Commons license and your intended use is not permitted by statutory regulation or exceeds the permitted use, you will need to obtain permission directly from the copyright holder. To view a copy of this license, visit http://creativecommons. org/licenses/by/4.0/.

(c) The Author(s) 2018 